\title{
An Image Transform Based on Temporal Decomposition
}

\author{
Felix Cremer ${ }^{\circledR}$, Mikhail Urbazaev, Christian Berger, Miguel D. Mahecha, \\ Christiane Schmullius, and Christian Thiel
}

\begin{abstract}
Today, very dense synthetic aperture radar (SAR) time series are available through the framework of the European Copernicus Programme. These time series require innovative processing and preprocessing approaches including novel speckle suppression algorithms. Here we propose an image transform for hypertemporal SAR image time stacks. This proposed image transform relies on the temporal patterns only, and therefore fully preserves the spatial resolution. Specifically, we explore the potential of empirical mode decomposition (EMD), a data-driven approach to decompose the temporal signal into components of different frequencies. Based on the assumption that the highfrequency components are corresponding to speckle, these effects can be isolated and removed. We assessed the speckle filtering performance of the transform using hypertemporal Sentinel-1 data acquired over central Germany comprising 53 scenes. We investigated speckle suppression, ratio images, and edge preservation. For the latter, a novel approach was developed. Our findings suggest that EMD features speckle suppression capabilities similar to that of the Quegan filter while preserving the original image resolution.
\end{abstract}

Index Terms-Multitemporal filtering, radar data, speckle, synthetic aperture radar (SAR).

\section{INTRODUCTION}

$\mathbf{I}$ N APRIL 2014 and in April 2016, the European Space Agency launched the new synthetic aperture radar (SAR) satellites Sentinel-1A and Sentinel-1B. Their sensors feature an enhanced radiometric and spatial resolution as well as a fundamentally improved temporal coverage compared with previous (C-band) SAR satellites. Imagery based on the same relative orbit is acquired with a time step of only six days. This acquisition strategy enables the acquisition of 60 images per year for a given region of interest. These new dense time series permit and require the development of new filtering and interpretation algorithms. However, SAR images are affected by speckle - a physical phenomenon inherent to coherent

Manuscript received July 6, 2017; revised September 28, 2017, November 7, 2017, and December 13, 2017; accepted December 26, 2017. Date of publication February 23, 2018; date of current version March 23, 2018. This work was supported in part by DLR in the Sentinel4REDD project under Grant FKZ:50EE1540, in part by the ARSAfricaE project under Grant FKZ:01LL1303D, and in part by the EU-H2020 program through the BACI project under Grant 640176. (Corresponding author: Felix Cremer.)

F. Cremer, M. Urbazaev, C. Berger, C. Schmullius, and C. Thiel are with the Chair of Earth Observation, Friedrich-Schiller University, 07743 Jena, Germany (e-mail: felix.cremer@uni-jena.de).

M. D. Mahecha is with the Max Planck Institute for Biogeochemistry, 07745 Jena, Germany.

Color versions of one or more of the figures in this letter are available online at http://ieeexplore.iee.org.

Digital Object Identifier 10.1109/LGRS.2018.2791658 imaging systems featuring a greater geometric resolution compared with the sensing wavelength [2]. In most remote sensing applications based on SAR imagery, speckle is considered as disturbing noise that should be reduced to obtain images allowing for classic and proven information extraction techniques. Therefore, speckle filtering is a common preprocessing step. A comprehensive overview of monotemporal speckle filters is given in [3]. The backscatter response of a resolution cell is influenced by geometric and dielectric properties of the surface [4]. If we are tracking one resolution cell in time, temporal changes of the geometric and dielectric properties cause variations of the speckle. For instance, changes of the geometric properties can be related to vegetation dynamics or human activities. The dielectric properties are mostly determined by the water content in the soil or the vegetation. The water content can change on different time scales, e.g., seasonally following hydrometeorlogical cycles, or at the monthly scale in response to, e.g., droughts, but may also reflect rapid rainfall pulses. Therefore, a pixel time series can be regarded as a superposition of different processes that are influencing the dielectric properties on different temporal frequencies (i.e., time scales). We can also expect that the resulting signals are typically nonstationary.

After the launch of the European Remote Sensing satellites in the 1990s, several techniques to use the multitemporal information were developed. One frequently used approach is the multitemporal filter by [1], which is a recursive version of the filter proposed in [5]. The filter computes local statistics in every band with a moving window approach. These local statistics are then multiplied with their temporal average. Therefore, the ordering of the time series is not considered. This approach leads to the same filtering statistics at each time step, which is a drawback for land-cover types with nonstationary temporally changing speckle behavior such as expected over agricultural areas. Our aim is to develop an image transform that solely operates in the time domain in order to fully preserve spatial details. Specifically, we explore the potential of using a variant of "empirical mode decomposition" (EMD) originally proposed in [6] as a filtering approach that additionally enables a suitable interpretation of the data. This letter is structured as follows. In Section II, we describe the temporal decomposition using EMD and define the filter settings. In Section III, we use Sentinel-1 data to compare the results of this filter with the results of the multitemporal speckle filter after [1]. Section IV discusses the findings and explores its potential for future applications. 


\section{Method}

The temporal backscatter signal is the superposition of signals with differing frequencies. The main idea of the image transform is to decompose the temporal backscatter information of each pixel in the logarithmic scale into these different frequency domains. For this, we use the EMD, which is suitable to handle nonstationary data. This method was developed in [6] and improved in [7] as the complete ensemble EMD (CEEMD). It can be understood as a generalization of the discrete Fourier transformation, as it also decomposes the signal into parts of different frequencies. Unlike a Fourier or wavelet transform, however, EMD does not rely on an a priori defined set of base functions. Rather, the EMD approach decomposes a time series in a fully dataadaptive manner. The retrieved partial signals are "intrinsic mode functions" (IMFs) and can show moderate phase and amplitude modulations, which makes the method very suitable for short and nonstationary time series. The IMFs are defined to have an equal number of zero crossings and local extrema and an average of the splines through the local minima (respectively, maxima) of zero.

The algorithm to compute the IMFs works as follows.

1) Detect the local extrema of the original data set $x(t)$.

2) Compute one spline through the local minima $\left(s_{\min }\right)$, and one through the maxima $\left(s_{\max }\right)$, respectively.

3) Compute the mean of $s_{\min }$ and $s_{\max }$ and subtract it from $x(t)$

$$
h_{1, k}(t)=x(t)-\frac{s_{\min }-s_{\max }}{2} .
$$

4) Repeat the previous steps with $h_{1, k}$ as $x(t)$ until the numbers of zero crossings and local extrema of $h_{j, k}$ are equal. Then $h_{j, k}$ is the preliminary IMF $c_{1}(t)$ of $x(t)$.

5) Repeat steps 1-4 with added Gaussian white noise and employ the average of the different representation as $\mathrm{IMF}_{l}$.

6) Repeat steps 1-5 with the difference between $x(t)$ and $\mathrm{IMF}_{l}$ until there is no IMF to extract, i.e., the remaining signal has at most one extremum.

This procedure decomposes the data set $x(t)$ into $N$ IMFs $c_{i}$ and one residual $r$

$$
x(t)=\sum_{i=1}^{N} c_{i}(t)+r(t) .
$$

We use the implementation of CEEMD implemented by [8] in C. This package has python bindings available at [9].

At the beginning and the end of the time series, the algorithm cannot decide if the data are a local minimum or maximum. Accordingly, the filter needs an initialization phase and a small number of images at the edges of the time series must be discarded.

Fig. 1 shows the decomposition of the Sentinel- 1 time series of a $3 \times 3$ matrix of broadleaved forest pixels. The frequency is decreasing for increasing IMF numbers. The IMF 0 and IMF 1 are high-frequency components. In contrast, for the higher IMF numbers such as IMF 2 and IMF 3 , the temporal behavior of the nine neighboring pixels becomes similar. This indicates

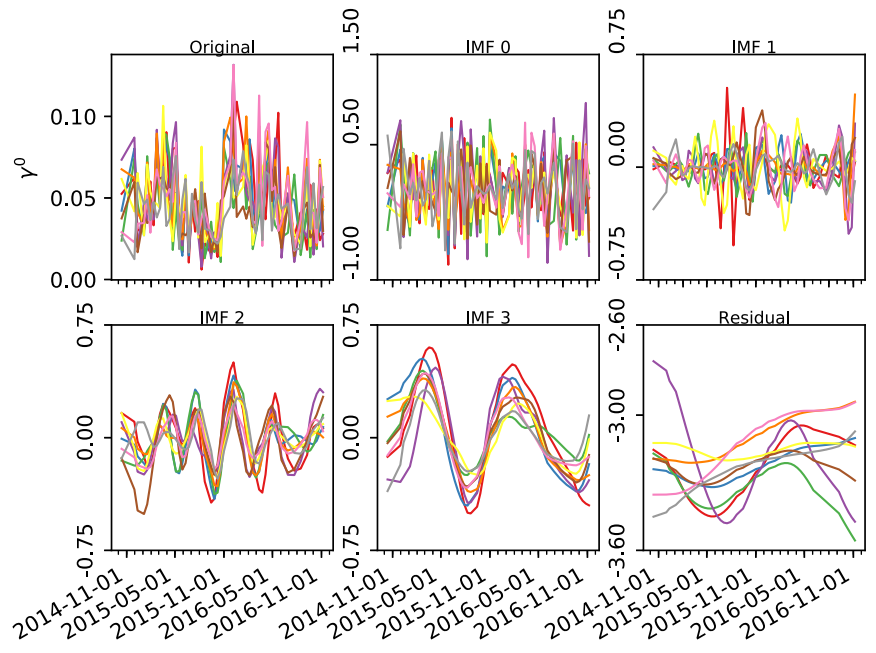

Fig. 1. Decomposition of the time series of a $3 \times 3$ matrix into the IMFs. All graphs correspond to broadleaved forest.

that these higher IMF numbers are less affected by speckle but driven by similar biophysical processes in similar (adjacent) resolution cells. IMF 2 features a periodicity of half a year and IMF 3 of one year. The residual of the IMF process is related to the overall trends.

According to the IMF behavior, we can assume that speckle mainly impacts the IMFs representing the higher frequencies of the temporal signal. IMFs with lower frequencies are related to biophysical processes. Thus, for the transformation of the image, the two IMFs with the highest frequencies are removed. Note, however, that also short-term extrema such as increased backscatter after a rainfall event will be filtered, which can be a desired side effect for many applications.

\section{EXPERIMENTAL RESULTS}

\section{A. Data and Preprocessing}

The proposed method was tested on a stack of 53 Sentinel-1 GRD images of the same relative orbit collected over Central Germany (close to Erfurt) at flat terrain (mean slope $3.44^{\circ} \pm$ $3.38^{\circ}$ ) between October 2014 and November 2016. The time span between the acquisitions was 12 days for most of the images. Exceptions include a gap of 48 days at the beginning of the time series and eight gaps of 24 days. Fig. 2 shows one image of the temporal stack.

The data have been preprocessed using the GAMMA software [10]. The single time steps are multilooked to a $10 \mathrm{~m} \times 10 \mathrm{~m}$ pixel spacing. The orthorectification is based on the original orbit state vectors and the 30-m SRTM digital elevation model [11]. The preprocessing also included radiometric terrain flattening after [12], which results in $\gamma^{0}$ backscatter values. All images were coregistered in the DEM geometry after geocoding to achieve a subpixel coregistration precision that is of eminent importance when the pixels are investigated in the temporal domain only.

\section{B. Evaluation of Speckle Reduction}

We compare the results of our approach with the results derived by the Quegan filter using $3 \times 3$ and $5 \times 5$ spatial windows. Fig. 3 shows the results of the different filters in a 


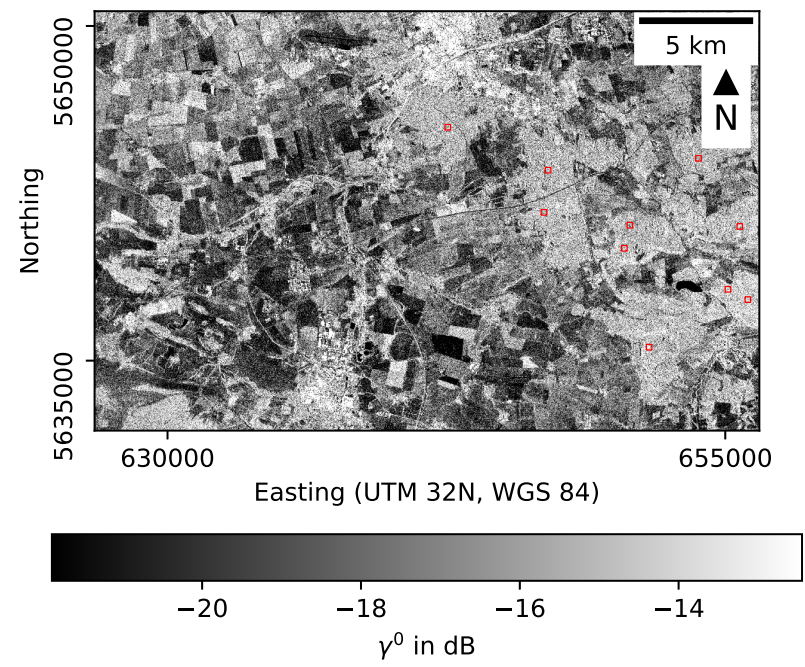

Fig. 2. Study area near Erfurt. The northeast of the image contains mixed forest. The straight lines in the southeast and the curve in the center are highways. The dark and bright rectangles are agricultural fields. This Sentinel-1 image was acquired at September 8, 2015 and has a resolution of $10 \mathrm{~m}$. The red squares indicate the homogeneous forest areas for the computation of the ENL.

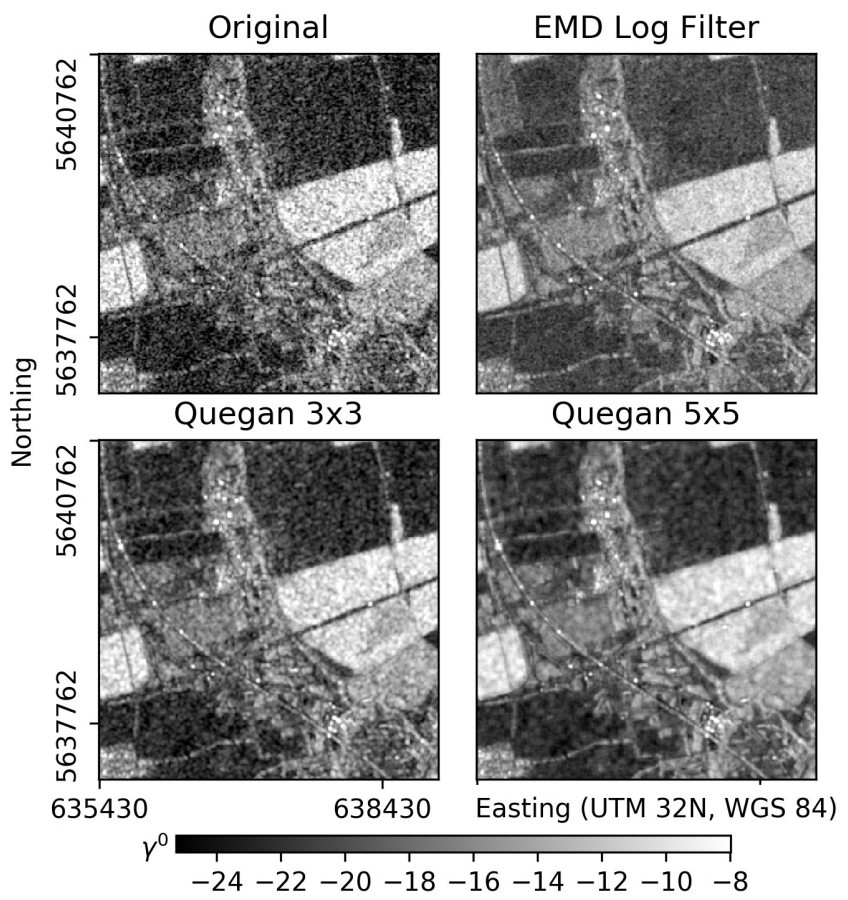

Fig. 3. Comparison of the different filters on Sentinel-1 data acquired on October 6, 2015. It is visible that all filters reduce the speckle in homogeneous areas. In the middle of the image, the highway crossing is better distinguishable in the transformed image.

subset of the study area. Visually, forest areas and agricultural fields are apparently smoothed in all filtered images. However, linear features such as highways, rails, or edges between different land-cover types appear clearer with the EMD filter.

We compute the equivalent number of looks (ENL) for every filter approach to compare the level of smoothing in homogenous areas. The ENL was defined in [13] and corresponds to the number of looks, which would be needed to obtain the same level of smoothing via multilooking. The ENL is

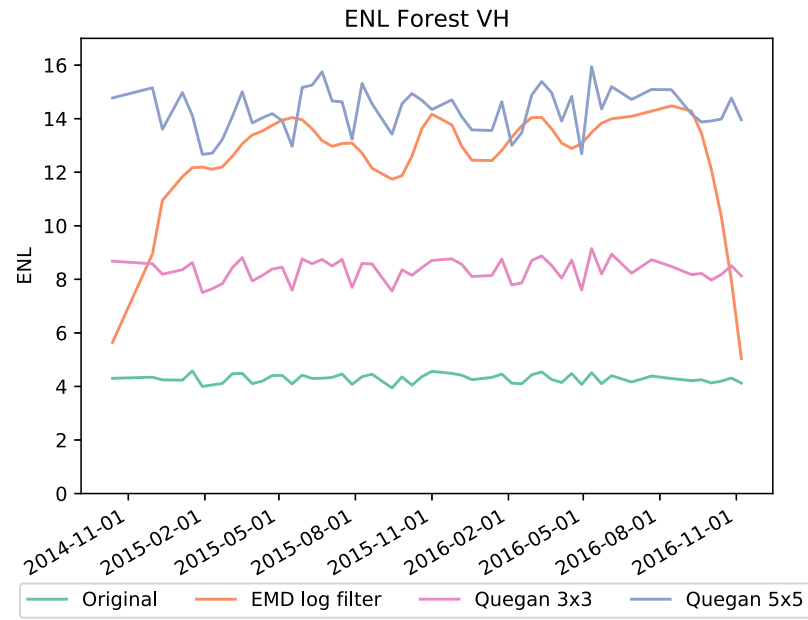

Fig. 4. Averaged ENL of 10 forest samples over time. The proposed filter has equal smoothing capabilities as the Quegan filter with a $5 \times 5$ spatial window.

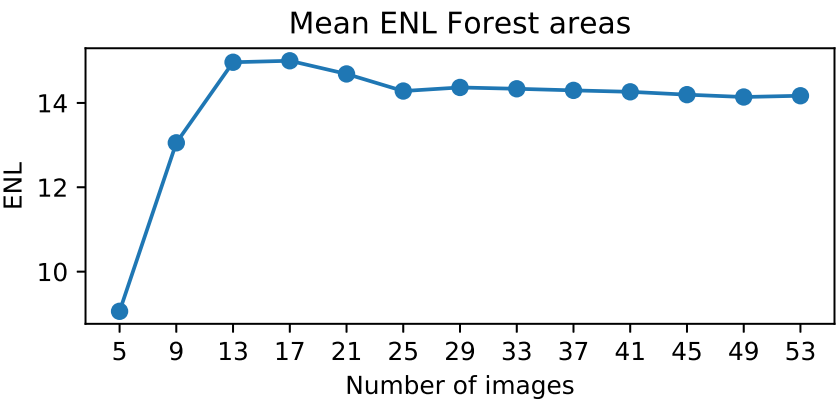

Fig. 5. ENL of the proposed method with different time series lengths. The ENL is computed over the same areas as in Fig. 4. The transform stabilizes with more than 13 images.

defined as

$$
\mathrm{ENL}=\frac{\mu\left(\hat{f}_{i}\right)^{2}}{\operatorname{Var}\left[\hat{f}_{i}\right]}
$$

Hereby $\hat{f}_{i}$ is the filtered image in a homogenous area at time step $i$ and $\mu$ and Var are the mean and the variance.

For this computation, we defined 10 homogeneous samples of forest areas. The positions of the samples are shown in Fig. 2. Each sample has a size of $25 \times 25$ pixels $\left(6.25 \mathrm{~km}^{2}\right)$. Fig. 4 shows the averaged ENL for the forest samples. The ENL of the original image fluctuates between four and five, which is the expected value according to the multilooking. The noise reduction of the EMD filter is improved against the $3 \times 3$ Quegan filter and comparable with the $5 \times 5$ Quegan.

The dropping of the ENL for the EMD Filter at the temporal edges is one of the characteristics of this approach that requires an initialization phase. To determine the length (number of images) of the initialization phase, the EMD filter was applied to time stacks of different lengths (Fig. 5). For this, the ENL is computed for the acquisition of October 20, 2015. The temporal stack was stepwise extended by concatenating Sentinel-1 images at both temporal sides of the stack. The ENL stabilizes when a number of approximately 13 images are reached. Accordingly, six images should be discarded from both sides of the time series to avoid less efficient filtering due to the initialization phase. 

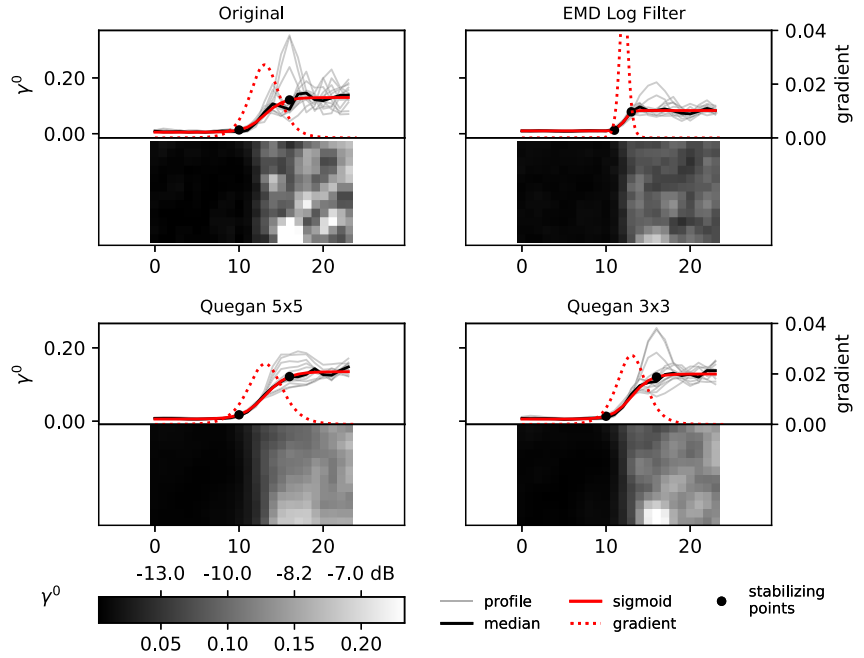

Fig. 6. Comparison of an edge between different crops on October 6, 2015 in different speckle filters. The median of the lines orthogonal to an assumed edge is approximated by a sigmoid function. The black points indicate the width of the edge as a measure of the blurring effect and the red dotted line indicates the slope of the sigmoid function.

\section{Evaluation of Edge Preservation}

The assessment measures of the edge preservation are mostly based on the use of reference images (see [3] for a discussion of measures). The only measure that is suitable for real SAR images is the edge preservation index by [14]. This approach is, however, not robust in the sense that: 1) edge detection algorithms are not usable on unfiltered SAR images and 2) the subjectivity of manual edge localization.

We therefore propose a novel approach for assessing edge preservation. Fig. 6 shows an edge between two agricultural fields with different crops. The SAR data were filtered using the Quegan filter and the EMD filter. The map shows a $24 \times 12$ pixel subarea comprising the edge. The gray lines in Fig. 6 show the pixel values as lines orthogonal to the edge. The black line is the median parallel to the edge in every pixel. To determine the position and magnitude of the edge, we fit a sigmoid function depicted in red. We use the generalized logistic function defined by [15]

$$
f(x)=l+\frac{u-l}{\left(1+q * e^{-g *(x-M)}\right)^{\frac{1}{v}}}
$$

where $x$ denotes the pixel position, $l$ and $u$ are the lower and upper bounds, and $g$ is the growth rate. The parameter $M$ moves the function in the $x$-direction and $v$ and $q$ finetune the length and position of the slope. The edge is located at the inflection point of the sigmoid function. The width of the edge is indicated by the position of the extrema of the third derivative of the sigmoid function. By the use of the fitted sigmoid function, small deviations in the backscatter are not influencing the assessment of the wideness of the edge. According to the results, it can be concluded that EMD filter improves the edge between the two fields, while the Quegan filter tends to introduce blurring. The edge preservation evaluation was applied to further acquisitions of the time series (Tables I and II). According to the results, an improved
TABLE I

LENGTH of THE INCLine of THe Detected Edge. A LoW LENGTH OF THE INCLINE INDICATES A SHARP EDGE

\begin{tabular}{l|c|c|c|c} 
Aquisition date & Original & EMD transform & Quegan 3x3 & Quegan 5x5 \\
$2015-05-29$ & 2 & 2 & 2 & 2 \\
$2015-06-10$ & 6 & 2 & 6 & 6 \\
$2015-06-22$ & 3 & 2 & 6 & 6 \\
$2015-07-04$ & 2 & 2 & 2 & 6
\end{tabular}

TABLE II

High SLOPE INDiCATES A High BACKSCATTER DIFFERENCE IN THE NEIGHBORING FIELDS

\begin{tabular}{l|c|c|c|c} 
Aquisition date & Original & EMD transform & Quegan 3x3 & Quegan 5x5 \\
$2015-05-29$ & 0.107 & 0.041 & 0.043 & 0.034 \\
$2015-06-10$ & 0.027 & 0.056 & 0.027 & 0.024 \\
$2015-06-22$ & 0.029 & 0.041 & 0.018 & 0.016 \\
$2015-07-04$ & 0.563 & 0.023 & 0.028 & 0.013
\end{tabular}

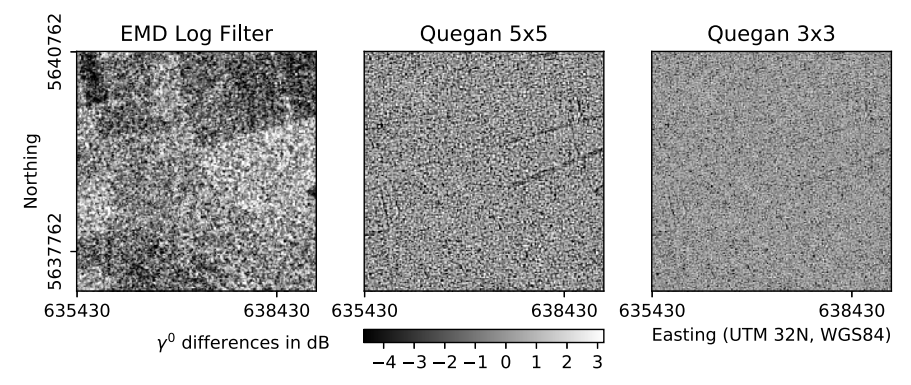

Fig. 7. Ratio images of the different filters converted to decibel differences.

edge preservation can be observed compared with the Quegan filter.

\section{Ratio Images}

For a further assessment of the performance of the EMD filter, ratio images of despeckled and original images were computed. The ratio of the despeckled image with the original

$$
r_{\text {speckle }}=\frac{I}{\hat{I}}
$$

should be white noise only because of the multiplicative noise assumption. The visibility of other features indicates spatial blurring or temporal mixing of the filtered data. Fig. 7 shows examples of such ratios.

For the Quegan filter, the ratio basically corresponds to white noise. Nevertheless, some linear features are visible. In the ratio of the EMD filter, no linear structures appear. However, in contrast to the Quegan filter, structures related to agricultural fields are clearly visible. This indicates that extreme temporal peaks appearing in one or two chronologically adjacent images are smoothed by the proposed image transform. These temporary outliers of backscatter can be related to various environmental impacts such as heavy precipitation events, flooding, frost, or special scattering processes of a specific crop at a particular growing stage. For applications focusing on those special events, the proposed filter might result in decreased detection rates. However, most application such as land-cover monitoring, change detection, 
or degradation monitoring shall benefit from the filtering of these backscatter outliers.

\section{DISCUSSION}

The use of multitemporal radar images has been shown to be an effective method to filter speckle in SAR imagery [1], [16]-[18]. However, in contrast to [1], in this letter, we filtered the SAR imagery in time domain only, which preserves the spatial resolution. This is of great importance for applications that require high geometric resolution such as forest degradation monitoring. Our results showed similar statistical results compared with the Quegan filter in terms of speckle suppression and an improved edge preservation. According to the experiment, ENL values of up to 15 can be achieved over forested areas. If further smoothing is needed, the proposed transform could be combined with a monotemporal speckle filter (see [13]). The proposed transform also removes short-term deviations (appearing in only one or two consecutive images) of backscatter that can be related to specific environmental conditions. Depending on the aim of the SAR data usage, this characteristic of the EMD filter can be beneficial. If the SAR data application aims at the detection of short-term extreme events, monotemporal filters such as [13] are preferable.

\section{CONCLUSION}

To the best of our knowledge, this letter presents the first approach of using dense SAR time series (up to 60 acquisitions per year) to filter speckle in SAR imagery in the time domain only. Exploring novel approaches of this kind is highly relevant to enable the usage of Sentinel-1 satellites. The filter is based on the EMD, such that each pixel is separately decomposed into intrinsic mode functions (IMFs) of different temporal frequencies. In order to reduce the speckle, the two IMFs with the highest frequencies are removed. This results in a nonparametric image transform that fully preserves the geometric resolution. The test results show that the speckle suppression is comparable with the Quegan filter. We developed a new method to measure edge preservation by approximating the width and slope of an edge. This showed that the EMD filter has a better edge preservation than the Quegan filter. Ratios of the filtered and original images show that the EMD filter removes not only the speckle but also temporal outliers.

The conservation of the full geometric resolution might open new opportunities for applications requiring high spatial resolution. One prominent example is forest degradation mapping in the context of the Reducing Emissions from Deforestation and Forest Degradation (REDD+) initiative. Future work will address the use of 2-D or 3-D EMD in order to combine temporal and spatial filtering to further improve the realizable smoothing. This new image transform will be examined for deforestation and degradation mapping.

\section{ACKNOWLEDGMENT}

This work uses Copernicus Sentinel data 2014-2016. The authors would like to thank the reviewers for their valuable comments and suggestions to improve the quality of this letter.

\section{REFERENCES}

[1] S. Quegan and J. J. Yu, "Filtering of multichannel SAR images," IEEE Trans. Geosci. Remote Sens., vol. 39, no. 11, pp. 2373-2379, Nov. 2001.

[2] I. H. Woodhouse, Introduction to Microwave Remote Sensing. Boca Raton, FL, USA: CRC Press, 2006.

[3] F. Argenti, A. Lapini, T. Bianchi, and L. Alparone, "A tutorial on speckle reduction in synthetic aperture radar images," IEEE Geosci. Remote Sens. Mag., vol. 1, no. 3, pp. 6-35, Sep. 2013.

[4] M. C. Dobson, F. T. Ulaby, and L. E. Pierce, "Land-cover classification and estimation of terrain attributes using synthetic aperture radar," Remote Sens. Environ., vol. 51, no. 1, pp. 199-214, 1995.

[5] J. Bruniquel and A. Lopes, "Analysis and enhancement of multitemporal SAR data," Proc. SPIE, Image, Signal Process. Remote Sens., vol. 2315, pp. 342-353, Dec. 1994, doi: 10.1117/12.196733.

[6] N. E. Huang et al., "The empirical mode decomposition and the Hilbert spectrum for nonlinear and non-stationary time series analysis," Proc. Roy. Soc. London A, Math., Phys. Eng. Sci., vol. 454, no. 1971, pp. 903-995, Mar. 1998.

[7] M. E. Torres, M. A. Colominas, G. Schlotthauer, and P. Flandrin, "A complete ensemble empirical mode decomposition with adaptive noise," in Proc. IEEE Int. Conf. Acoust., Speech Signal Process. (ICASSP), May 2011, pp. 4144-4147.

[8] P. J. J. Luukko, J. Helske, and E. Räsänen, "Introducing libeemd: A program package for performing the ensemble empirical mode decomposition," Comput. Stat., vol. 31, no. 2, pp. 545-557, 2016.

[9] P. Luuko. (Jun. 2017). Pyeemd-A Python Interface to Libeemd. [Online]. Available: https://bitbucket.org/luukko/pyeemd

[10] U. Wegmüller, C. Werner, T. Strozzi, A. Wiesmann, O. Frey, and M. Santoro, "Sentinel-1 support in the GAMMA software," Procedia Comput. Sci., vol. 100, pp. 1305-1312, Jan. 2016.

[11] NASA Jet Propulsion Laboratory. NASA Shuttle Radar Topography Mission United States 1 arc second. Version 3. $50{ }^{\circ} \mathrm{N}, 11^{\circ} \mathrm{E}$. NASA EOSDIS Land Processes Distributed Active Archive Center (LP DAAC), U.S. Geol. Surv. Earth Resources Observation and Science (EROS) Center, Sioux Falls, SD, USA. Accessed: 2013. [Online]. Available: http://dx.doi.org/10.5067/MEaSUREs/SRTM/SRTMUS1.003

[12] D. Small, "Flattening gamma: Radiometric terrain correction for SAR imagery," IEEE Trans. Geosci. Remote Sens., vol. 49, no. 8, pp. 3081-3093, Aug. 2011.

[13] J.-S. Lee, "Speckle analysis and smoothing of synthetic aperture radar images," Comput. Graph. Image Process., vol. 17, no. 1, pp. 24-32, 1981.

[14] H. Chumning, G. Huadong, and W. Changlin, "Edge preservation evaluation of digital speckle filters," in Proc. IEEE Int. Geosci. Remote Sens. Symp., vol. 4. Jun. 2002, pp. 2471-2473.

[15] F. J. Richards, "A flexible growth function for empirical use," J. Experim. Botany, vol. 10, no. 2, pp. 290-301, 1959.

[16] E. Trouvé, Y. Chambenoit, N. Classeau, and P. Bolon, "Statistical and operational performance assessment of multitemporal SAR image filtering," IEEE Trans. Geosci. Remote Sens., vol. 41, no. 11, pp. 2519-2530, Nov. 2003.

[17] M. Ciuc, P. Bolon, E. Trouvé, V. Buzuloiu, and J.-P. Rudant, "Adaptive-neighborhood speckle removal in multitemporal synthetic aperture radar images.," Appl. Opt., vol. 40, no. 32, pp. 5954-5966, Nov. 2001.

[18] T. T. Lê, A. M. Atto, and E. Trouvé, "Adaptive multitemporal SAR image filtering based on the change detection matrix," IEEE Geosci. Remote Sens. Lett., vol. 11, no. 10, pp. 1826-1830, Oct. 2014. 